\title{
Pore Evolution and Channel Formation During Flow and Reaction in Porous Media
}

A theoretical and experimental study on the dissolution of porous media by flowing acid has been carried out. Dissolution of the media results in an evolution of the pore geometry and the formation of random flow channels. The goal is to predict the range of conditions under which channels will form, and the effects of various parameters on the structure of the channels and on their rate of propagation through the media. A random network model is used to describe the behavior of the stochastic, rootlike channels that form during flow and dissolution in carbonate rock and in other systems. The structure of the flow channels that form as a result of acid attack are characterized and studied using a Wood's metal casting technique. A comparison of model results shows that the rates of channel formation and growth are intimately related to the developing structure of the channels (size of branches and degree of branching), which in turn is controlled by factors such as the fluid velocity and the rate of reaction. Depending on the experimental conditions, the channels range from a single conduit with a minimum of branching to a highly branched, spongy network of channels. The dependence of permeability increase and channel branchedness on injection rates and acid diffusion rates is described by the Damkohler number for flow and reaction.

\section{Introduction}

The chemical reaction between a fluid and a porous medium through which it flows is an area of fundamental and practical interest. As the medium is dissolved by reactant in the fluid, flow channels are etched in the porous solid. The structure and the behavior of dominant channels that form depend on the local flow conditions and on the reaction rate. Understanding the channeling process is necessary in order to predict the movement of the reaction or dissolution zone through the porous medium.

This study has important applications in processes designed to enhance oil and gas production from petroleum reservoirs. These processes, referred to collectively as stimulation treatments, involve injecting acid into wells with the intent of dissolving some of the porous rock near the wellbore, thus increasing its permeability, or capacity for flow. Channels resulting from the dissolution serve to carry acid into the formation during the stimulation and also provide a lower resistance for the flow of oil from the formation after the stimulation is completed.

The flow and reaction in porous media described here can take place by one of two mechanisms. In one case, reaction between the fluid and the solid matrix takes place relatively uniformly as the reaction front moves through the porous medium. The corresponding permeability increase and other effects are likewise uniform throughout the medium. An example of this dissolution mechanism is the hydrofluoric acid stimulation of sandstone. The second dissolution mechanism involves channeling, and this is the area of primary focus in this work. Channeling involves a highly selective pattern of dissolution that results in the formation of a random network of channels. The effect of the dissolution process in this case is not uniform because only certain parts of the medium are permeated by channels, while others can remain virtually unaffected. Channeling occurs when all of the matrix material that is present can dissolve, for example, the dissolution of porous carbonate rock by hydrochloric acid. The inert portion of the matrix in sandstone, however, prevents any such channeling from occurring.

\section{Pore evolution leading to channel formation}

In studying channeling, or "wormholing," as it is referred to in the petroleum literature, one wants to discover how the reac- 
tive fluid flows through the medium when simultaneous mineral dissolution and evolution of the pore structure occurs. An example of this channeling phenomenon in limestone is shown in Figure 1.

Several studies have addressed the effects of flow and dissolution in carbonates (Nougaro and Labbe, 1955; Rowan, 1959; Nierode and Williams, 1971; Guin et al., 1971), all observing that permeability increases occur as a result of the highly branched channels, or wormholes, that form as a result of dissolution. These studies have not, however, been able to relate the effects of parameters such as the fluid velocity, the heterogeneous reaction rate, or the properties of the media, to the channel structure, the rate of propagation, and the increases in permeability. This previous lack of understanding is due primarily to the stochastic nature of channel formation and growth and to the inherent difficulty in modeling the random phenomena. In this work, the goal is to relate the effects of the above parameters to the structure of the network of channels that form, to the rate of propagation of the channels, and to the resulting overall increase in permeability of the media.

\section{Channeling in Porous Media \\ Mechanism of channel formation}

The structure of channels that can form in porous media as a result of flow and dissolution can be very similar to patterns associated with diffusion-limited aggregation produced by a variety of other processes (Witten and Sander, 1981, 1983; Meakin, 1983; Paterson, 1984). The channeling resulting from dissolution is also similar to viscous fingering observed during miscible or immiscible floods (Chen and Wilkinson, 1985; Daccord et al., 1985; Paterson, 1984). In both these cases, the flow systems are unstable to perturbations in the fluid front that result primarily because of the heterogeneity of the porous media.

In the dissolution system, perturbations in the rate of dissolution occur in a small section of an initially uniform reaction profile (front). These perturbations result in some of the acid advancing ahead of the average position of the reaction front and dissolving material ahead of the front. This dissolution

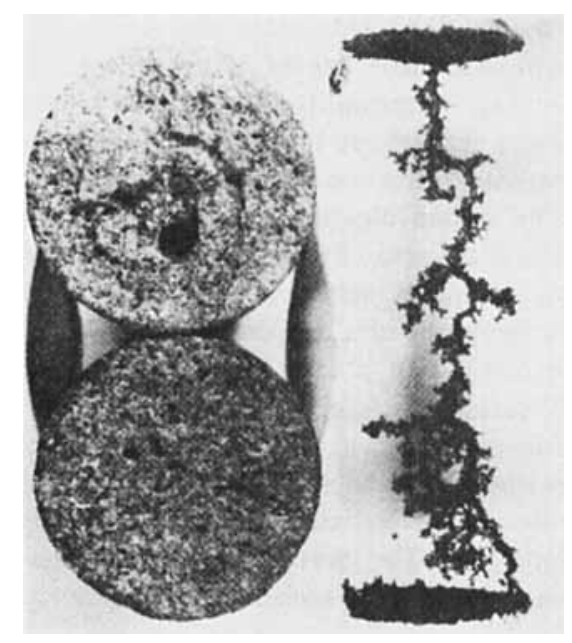

Figure 1. Example of channeling in porous limestone.

Inlet (top) and outlet flow faces of a cylindrical core $(0.38 \mathrm{~m}$ dia. $\times$ $0.127 \mathrm{~m}$ length Indiana limestone) treated with aqueous $\mathrm{HCl}$, along with a Wood's metal wormhole casting reduces the resistance to acid flow and consequently more acid contacts this area, producing an even greater amount of dissolution in the area of the initial perturbation. Soon, a channel forms and continues to grow because the resistance to flow in the channel is negligible compared to the resistance through the pore space that has not been affected by the acid. As these channels propagate, flow from other regions is diverted into dominant channels, leaving certain areas unaffected.

The channel growth rate is limited by the amount of acid that reaches the reaction front (i.e., the advancing channel tip). Therefore, the rate of propagation and the position of the reaction front are a function of the rate of reactant (acid) consumption between the inlet of the media and the front. To illustrate this we consider a linear core of porous material through which acid is flowing in the axial direction. If the reaction is very slow relative to the reactant flow rate then the dissolution rate will be uniform throughout the length of the core and all pore pathways will be dissolved. On the other hand, if reaction is fast relative to the flow, acid is consumed immediately upon entering the medium. As a result, the reaction front will not propagate because very little unreacted acid penetrates to the channel tips and only the face of the core will be dissolved. Since the structure of the medium behind the advancing channel tip is continuously dissolving and evolving, the acid concentration and the rate at which acid reaches the tip is continually changing. Consequently, the dissolution rate of the solid matrix is intimately related to the evolving pore structure.

The theory of diffusion-limited aggregation (DLA) has been used to describe viscous fingering, and is also being applied to flow and dissolution in certain model systems (Daccord, 1987; Daccord and Lenormand, 1986). DLA aggregates, which are essentially randomly generated clusters, have been able to reproduce the basic patterns produced by viscous fingering and dissolution. However, at present this theory cannot account for all the kinetic and mass transfer effects in a process such as acid dissolution. As stated previously, the rate of movement of the reaction front is limited by the transport of reactant to the front. This in turn is controlled by the relative rates of convective transport and chemical reaction. DLA simulations of model systems involving plaster dissolution in water predicted a constant rate of frontal advance. This type of behavior has not been observed in porous rock dissolution due to these kinetic effects. In reality, the rate of frontal advance must continually decrease with time for reasons that will be outlined here.

In this work, we look at the fundamental mechanisms leading to channeling in porous media, and at the properties or conditions that affect the channel behavior. Furthermore, we predict not only the structure of the channel network, but more important, the rate of propagation of the channel as a function of system parameters such as the fluid velocity and the reaction rate.

\section{Experimental Method \\ Coreflood experiments}

Porous cylindrical cores of limestone and dolomite of approximately 1 in. dia. and $4 \mathrm{in}$. length $(25 \times 100 \mathrm{~mm})$ were used in this study. The dissolution rates of limestone and dolomite differ sufficiently that it is possible to study two reaction-rate limiting regimes. Limestone dissolution, Eq. 1, is mass-transfer limited at temperatures above $0^{\circ} \mathrm{C}$ (Lund et al., 1973) while dolomite dissolution is reaction-limited below about $50^{\circ} \mathrm{C}$ (Lund et al., 1975). 
Limestone: $\mathrm{CaCO}_{3}+2 \mathrm{H}^{+}=\mathrm{CO}_{2}+\mathrm{H}_{2} \mathrm{O}+\mathrm{Ca}^{++}$

Dolomite: $\mathrm{CaMg}\left[\mathrm{CO}_{3}\right]_{2}+4 \mathrm{H}^{+}=2 \mathrm{CO}_{2}$

$$
+2 \mathrm{H}_{2} \mathrm{O}+\mathrm{Ca}^{++}+\mathrm{Mg}^{++}
$$

Several types of both limestone and dolomite cores were used, each exhibiting a range of initial permeabilities. Laboratory experiments were performed by first mounting a fluid-saturated core of either limestone or dolomite in the Hassler cell shown in Figure 2. When aqueous acid was used, the cores were saturated with pure water. When the microemulsion was used, a microemulsion identical to that containing the acid was used to saturate the core, except that this fluid contained water instead of acid. Acid was injected axially through the core at either a constant flow rate or a constant pressure drop. The permeability of the core was calculated as a function of the fluid volume injected by measuring either the pressure drop $\Delta P$ (constant rate injection), or the flow rate $Q$ (constant pressure drop injection) and using Darcy's law Eq. 3:

$$
\Delta P=Q \mu L / K A
$$

where $K$ is the permeability, $\mu$ the viscosity, and $A$ and $L$ the core cross-sectional area and length, respectively. A Ruska proportioning pump was used for the constant-rate studies to assure absolutely pulse-free fluid injection. Effluent $\mathrm{pH}$ and mineral concentration were monitored to track breakthrough of the reaction front at the end of the core. The experiments were conducted at room temperature and a minimum pressure of 1,000 psi $(6.89 \mathrm{MPa})$ was maintained in the core by a backpressure regulator downstream of the core. A high backpressure is required to maintain single-phase flow when gaseous reaction products are generated. Details of the experimental technique and the apparatus are described elsewhere (McCune et al., 1979).

Two acid systems were studied: aqueous $\mathrm{HCl}$ and an acid-inoil microemulsion system formulated to retard the rate of acid consumption (Hoefner and Fogler, 1985, 1987). The microemulsion was used to study the effects of the reaction rate in the transport-limited regime. No effects related to the oil/water/ surfactant system were observed other than the microemulsion exhibiting retarded acid diffusion rates. The acid concentrations used were 0.13 and $1.0 \mathrm{~N} \mathrm{HCl}$ for both the aqueous acid and the microemulsion. Bulk acid concentrations higher than $1.0 \mathrm{~N}$ were not used because of the excessive backpressure required to keep the higher $\mathrm{CO}_{2}$ concentrations in solution.

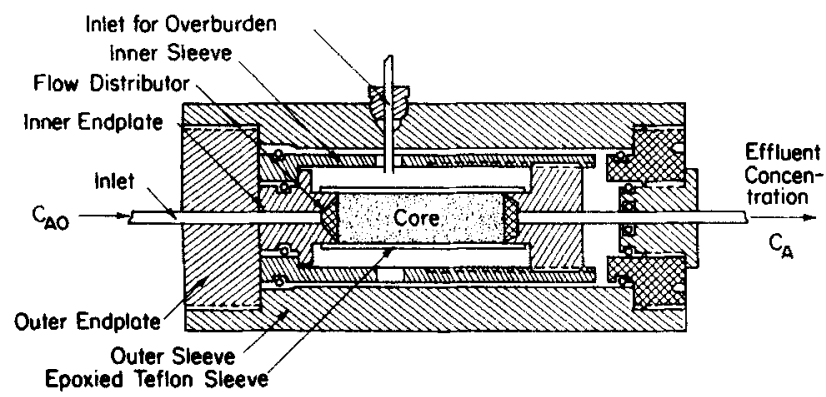

Figure 2. High-pressure Hassler flow cell used for linear flow experiments.

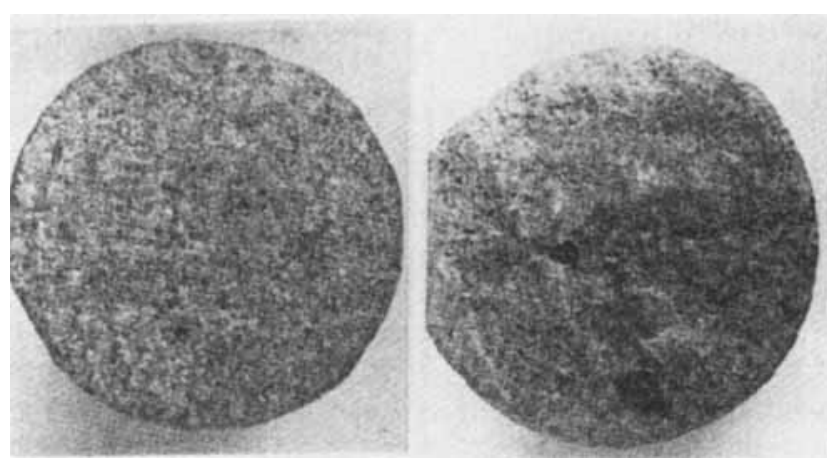

Figure 3. Inlet flow faces of limestone cores treated with aqueous $\mathrm{HCl}$ and with retarded acting microemulsion.

Note obvious channel formation in the aqueous core and more unjform penetration in the microemulsion core.

\section{Wood's alloy casting of wormholes in carbonates}

The wide variation in the size and number of wormholes visible on the core flow faces after treatment using aqueous acid and the microemulsion, Figure 3 , indicated that the fluid injection rate and the rate of acid attack significantly affect the wormhole structure. To study these effects, metal castings were made of the channels that formed in the porous rock by injecting molten Wood's alloy at $100^{\circ} \mathrm{C}$ into the channels and allowing the metal to solidify. The rock was then completely dissolved leaving a reproduction of the etched channels.

Wood's alloy is preferable over polymer resins for channel casting because it is nonwetting and, like mercury, it has a high surface tension. Thus the size of pores entered by the metal can be controlled by the injection pressure. This assures that only the new pore space resulting from mineral dissolution is invaded, and not the original pore space. In all cases, the cores were first evacuated and the metal was injected at atmospheric pressure in a vacuum oven.

\section{Discussion of Results}

\section{Coreflood results}

The permeability response of Indiana limestone cores acidized with aqueous $\mathrm{HCl}$ and with the microemulsion solution are shown in Figure 4. In this figure, the ratio of the permeability at any time to the initial permeability is plotted as a function of the number of pore volumes of fluid (acid) injected. The steep rise in

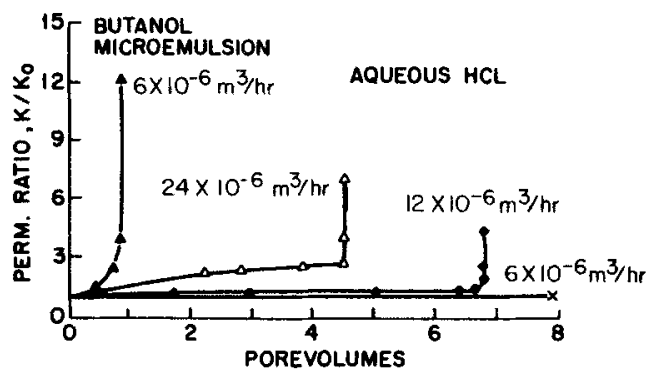

Figure 4. Coreflood results for Indiana limestone showing permeability response as a function of fluid injection rate.

$0.025 \mathrm{~m}$ dia. $\times .054 \mathrm{~m}$ length cores, $0.13 \mathrm{~N}$ aqueous $\mathrm{HCl}$ 
permeability is indicative of a dominant channel breaking through to the outlet flow face, at which point the resistance to flow across the core becomes negligible. The volume of fluid required to achieve breakthrough, which corresponds to the position of this steep rise in permeability, is referred to as the volume of fluid (acid) required to stimulate the core. The smaller the amount of acid required, the more efficient is the stimulation.

In runs made with limestone, the effluent acid concentration remains constant at about $\mathrm{pH}=5.5$ throughout the run and then suddenly drops to below $\mathrm{pH}=1$ exactly at the permeability breakthrough. This is indicative of a very steep acid concentration profile at the dissolution front, which is characteristic of a very fast reaction. For these runs, increasing the injection rate results in more efficient stimulation.

Wood's alloy castings of limestone cores treated using aqueous $\mathrm{HCl}$ and using $\mathrm{HCl}$ in a microemulsion are both shown in Figure 5. The castings are arranged in order of decreasing Damkohler number $D a$, which is the ratio of the net rate of reaction of acid with the pore wall to the rate of convective transport of the acid. The microemulsion formulation that was used exhibits an acid diffusion rate that is a factor of approximately 60 smaller than in aqueous $\mathrm{HCl}$. The castings clearly show how the channel structure evolves with changes in the Damkohler number. At large values of the Damkohler number, acid is essentially consumed completely at or near the injection face, Figure 6. At moderate values of the Damkohler number, live acid is carried further into the sample before reacting, and a channel forms. Further reduction in the Damkohler number results in increased channel branching, and the channels tend to be more uniform, spongy, and space-filling, representing more and more closely the structure of the original pore space, as observed on the righthand side of Figure 5.

Coreflood data for dolomite stimulated using aqueous $\mathrm{HCl}$ are shown in Figure 7 . In dolomite, where the dissolution rate is reaction-rate limited and is much slower than in limestone, a trend opposite to that seen in limestone is observed. The volume of fluid required to reach breakthrough in dolomite increases with decreasing Damkohler number. Acid is consumed over a

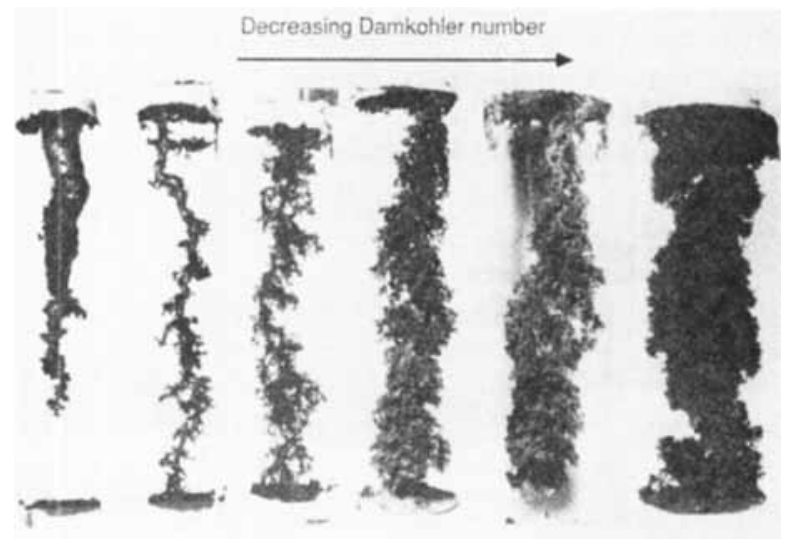

Figure 5. Wood's alloy castings of $0.127 \mathrm{~m}$ length cores treated under range of Damkohler number.

From left: aqueous $\mathrm{HCl}$ inject at $6 \times 10^{-6} \mathrm{~m}^{3} / \mathrm{h}$; aqueous $\mathrm{HCl}$ at $6 \times 10^{-5} \mathrm{~m}^{3} / \mathrm{h}$; microemulsion at $6 \times 10^{-6} \mathrm{~m}^{3} / \mathrm{h}$; aqueous $\mathrm{HCl}$ $6 \times 10^{-4} \mathrm{~m}^{3} / \mathrm{h}$; microemulsion at $6 \times 10^{-5} \mathrm{~m}^{3} / \mathrm{h}$; microemulsion at $6 \times 10^{-4} \mathrm{~m}^{3} / \mathrm{h}$.

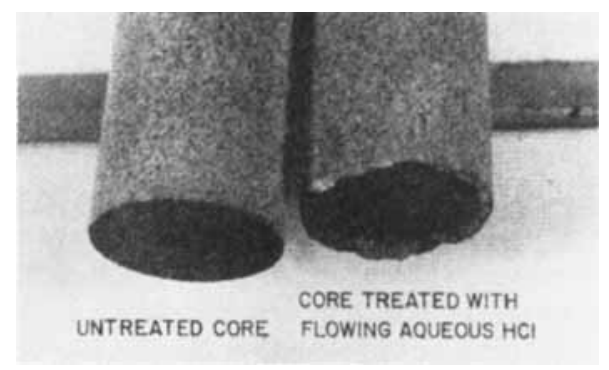

Figure 6. Inlet flow face of Indiana limestone core acidized with aqueous $\mathrm{HCl}$ at very low injection rate (right) and untreated core.

Note no acid penetration, but rather complete spending on flow face at the extreme of high Damkohler number

larger mineral surface area, which is exposed to live acid throughout owing to reaction limitations. Consequently, increasing the flow rate will not affect the dissolution rate but will increase the fluid volume necessary to break through the core. We would also expect very highly branched channels in the dolomite, and in fact we observe an almost uniform treatment of the pore space, as is shown in the dolomite casting in Figure 8.

In addition to the trends in the coreflood data, some general observations can be made about channel structure or branchedness from the channel castings. At high $D a$ numbers, the channel that forms is a single flow path with no branching. Close observation of the tip of the channel, however, reveals some very fine splitting of pathways that is typical of pore level branching. These very short branches do not appear anywhere else in the casting because they have been completely consumed within the very large diameter central channel.

Runs carried out at lower Damkohler numbers produce longer branches such as those shown in Figure 5. The number or density of these branches increases as the Damkohler number decreases. In general, the average length of a branch is short; branches up to about two core diameters in length have been observed, but this is rare. Finally, it has been observed that branches never rejoin and form closed loops. A good illustration of the range of branching that takes place can be seen in castings

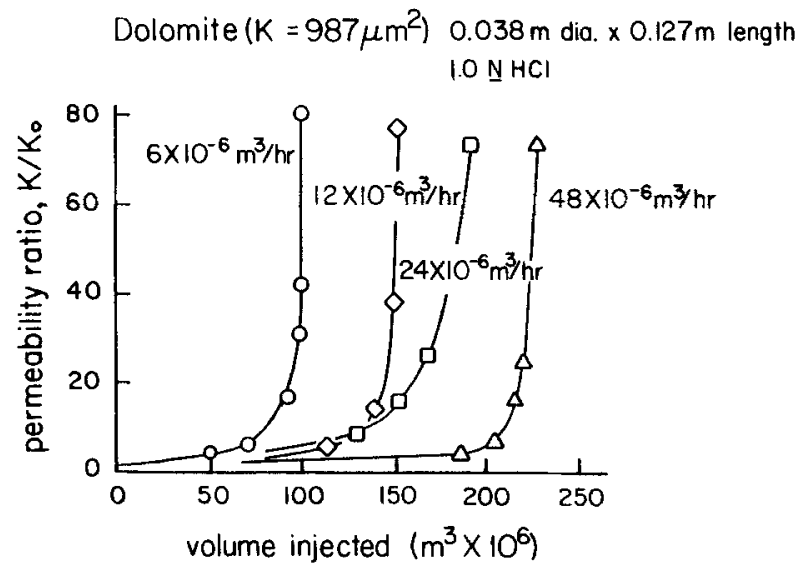

Figure 7. Coreflood data for dolomite showing permeability response as a function of fluid injection rate.

Note the opposite trend of that seen in Indiana limestone, Figure 4 


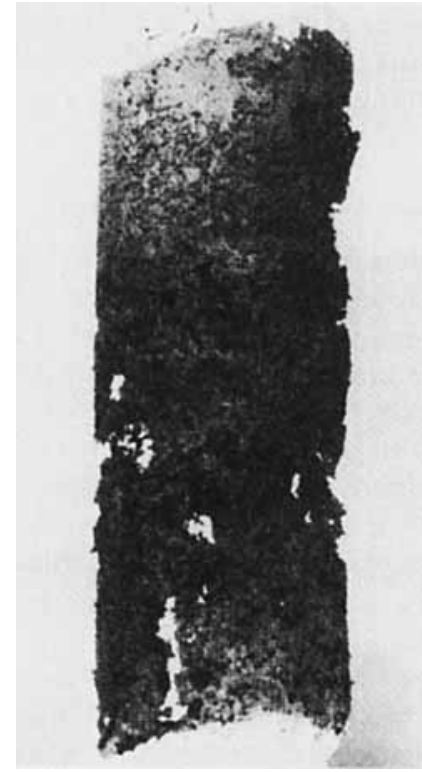

Figure 8. Wood's alloy casting of coreflood run in dolomite at a high injection rate.

The channeling in this case of extremely low Damkohler number tends to occupy a very large fraction of the original pore space

of coreflood experiments carried out at a constant pressure drop, Figure 9, where the Damkohler number varies from a very high value at the beginning of the run to a low value at the end. At the start of a run the flow rate is small because of the large resistance to flow, and a single channel is formed. However as the channel propagates through the core, the flow rate increases and the channels start to become finer and more highly branched.

A measure of channel branchedness can be obtained by measuring the surface area of the channel castings. If the branches are small in diameter and very numerous, the channel castings will exhibit a high surface area. On the other hand, single worm-

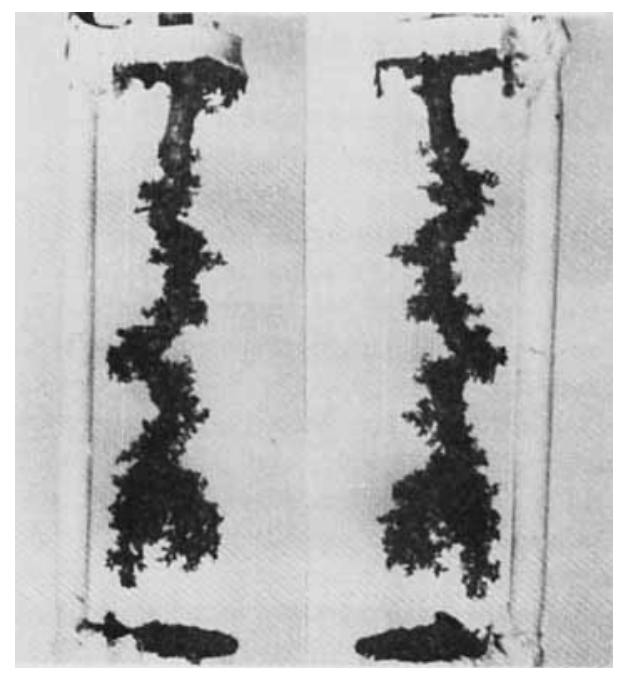

Figure 9. Wood's alloy casting of coreflood run at a constant pressure drop.

The flow rate increases throughout the run as the permeability is reduced, thus the casting shows the effects of a range of the Damkohler number holes will exhibit a low surface area per unit volume of channel. Figure 10 shows a general decrease in the area of the channels with increasing Damkohler number.

The permeability of the media has a large effect on channeling. This is especially true for the mass-transfer limited case where branching is more pronounced in higher permeability material for the same flow rate. Coreflood data for two types of limestone, Indiana limestone and Texas Cream chalk, have been compared and the higher permeability chalk requires more fluid to stimulate. Castings of the cores also show that at the higher injection rates branching is more pronounced in the chalk than in the Indiana limestone. This increase in branching can slow channel propagation. However, the effects of the higher fluid velocities attainable in the higher permeability rock will usually outweigh the effects of branching. Thus, in runs made at a constant pressure drop the higher permeability media can be stimulated more efficiently than lower permeability media.

\section{Development of the Network Model}

A random-network representation of the porous medium is used as the basis for a computer simulation to study channeling. The network approach was first introduced by Fatt (1956) and has been used by others since to describe processes in porous media where the important structural property of pore interconnectedness must be included. Examples where the network has been used successfully include fluid displacement (Simon and Kelsey, 1972), relative permeability (Rose, 1957), and dispersion in porous media (Sahimi et al., 1985, 1986a, b).

The network consists of a regular two-dimensional array consisting of nodes connected by bonds of cylindrical tubes. The triangular network (coordination number 6) shown in Figure 11 was used because lattices of this topology have been shown to exhibit certain properties (related to coordination number and connectedness) most like randomly generated two-dimensional Voronoi lattices (Jerauld et al., 1984). Fluid flow is simulated in the bonds, and mixing occurs in the nodes.

Before any calculations can be performed on the network, a distribution of resistances must be assigned. One would like to use the pore size distribution obtained from mercury injection because this is most easily obtained. There is, however, a large amount of ambiguity involved with interpreting mercury porosi-

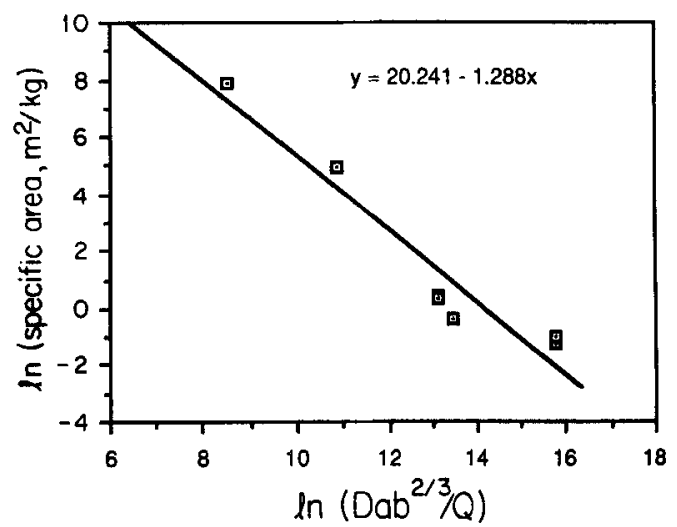

Figure 10. Plots of external surface area of castings as a function of Damkohler number.

The surface area is a measure of channel branchedness, with high area castings necessarily being highly branched, see Figure 5 


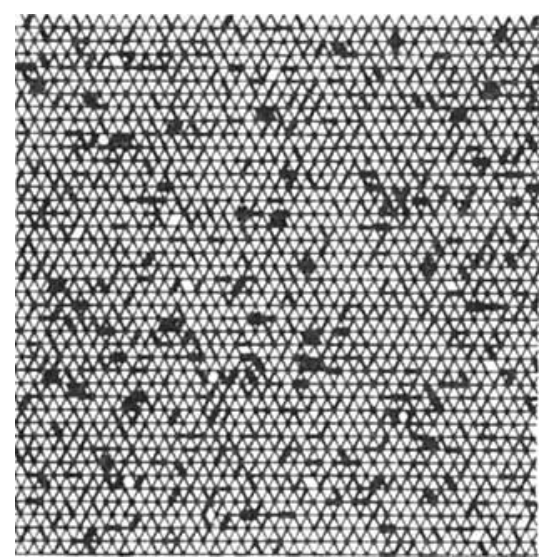

Figure 11. $40 \times 40$ two-dimensional triangular network decorated with a bond diameter distribution for Indiana limestone.

metry data. Literal interpretation of capillary data involves assigning pore size distributions assuming a bundle-of-tubes model for the porous medium. Unfortunately, this does not yield the true pore size distribution due to the effects of pore accessibility related to a percolation phenomenon that takes place during mercury injection (Fatt, 1956; Wardlaw and Taylor, 1976; Lapidus et al., 1985).

The approach in this study is to use a mercury injection simulation to estimate a realistic pore size distribution of the rock sample. A three-dimensional network is constructed, and mercury injection into the network is simulated. In this work the pore size distribution assigned to this three-dimensional network was varied until the shape of the simulated injection curve approximated the experimentally obtained mercury porosimetry curve for the sample considered. It is believed that this is the most realistic interpretation of simple mercury injection data. A network with coordination number 12 was used in the injection model, and injection was simulated from all faces of a $10 \times 10 \times$ 10 lattice representing about 6,000 pores.

Bond diameters following distributions of the form

$$
f(D)=k_{1} D^{\mathrm{cl}}\left(1-c 2 \cdot D^{\mathrm{c} 3}\right)
$$

where

$$
\begin{aligned}
f(D) & =\text { distribution function } \\
k_{1} & =\text { proportionately constant } \\
D & =\text { diameter }
\end{aligned}
$$$$
c 1, c 2, c 3=\text { constants }
$$

were found to be satisfactory to simulate the mercury injection data for Indiana limestone. This distribution was assigned over the diameter range $0.03-4.0 \mu \mathrm{m}$, which corresponds to the range of pressures over which mercury invaded Indiana limestone. Values of $c 1=-4, c 2=400$, and $c 3=2$ were assigned to the distribution. A network decorated with a typical distribution is shown in Figure 11.

Once the distribution is assigned to the bonds in the network, the flow through each bond in the network and the pressure at each node is calculated by simultaneously solving mass balances at each node for either constant pressure or constant injection conditions. The Hagen-Poiseuille relationship for laminar flow in cylindrical tubes is used to relate the flow rate and pressure drop in the individual bonds. A periodic boundary condition is assigned to the sides of the network. This representation of a porous medium is similar to previous investigations using the network model.

\section{Reaction and bond growth in the network}

This study differs from previous applications of the network model in that a reactant (acid) is introduced to simulate flow and dissolution, and the bonds are allowed to grow as they are contacted by the unconsumed acid. The fluid enters the network at the inlet flow face. Some of the acid is consumed immediately in the first bonds encountered, causing them to grow, while the remaining acid invades further to a distance where its concentration falls below some predetermined minimum. For the case of limestone dissolution in acid, this minimum concentration corresponds to the equilibrium $\mathrm{pH}$ of the particular acid in contact with $\mathrm{CaCO}_{3}$.

The rate of growth of a bond is a function of the net rate of reaction in that bond. Depending on the rate-limiting regime, the net rate of reaction is either the rate at which acid diffuses to the wall of a cylindrical bond or the rate of heterogeneous surface reaction with no mass transfer effects. Several general forms of the expression for the rate of acid depletion in the bonds have been considered. For the transport-limited case, either a general expression for transport in a long tube, or the Graetz solution, which is an exact solution to the laminar flow and diffusion, can be used. It is assumed for the mass-trancfer limited case that reactant concentration at the wall is o. For $\mathrm{L}_{2}$. reaction-limited case, the expression for reactant $c$ isumption is calculated using a plug-flow reactor model. The appropriate forms of the general expression, the Graetz solution, and a kinetic expression for the acid concentration, $C$, at a particular model location $L_{i}$ are given in Eqs. 5, 6, and 7, respectively (Jakob, 1949).

$$
\begin{gathered}
C_{i}\left(L_{i}\right)=C o_{i} \exp \left[-a D_{e}^{2 / 3} L_{i} /\left(Q_{i}^{1-b} D_{i}^{b} v^{b-1 / 3}\right)\right] \\
C_{i}\left(L_{i}\right) \sim C o_{i} \exp \left[-a\left(D_{e} L_{i} / Q_{i}\right)\right] \\
\left.C_{i}\left(L_{i}\right)=C o_{i} \exp \left[-a k L_{i} D_{i} / Q_{i}\right)\right]
\end{gathered}
$$

Equations 5-7 show the dependence of the rate of reaction on parameters such as the diffusion coefficient $D_{e}$, the specific reaction rate $k$, the specific the flow rate $Q_{i}$, the bond diameter $D_{i}$ and bond length $L_{i}$, the kinematic viscosity $v$, and on the constants $a$ and $b$. The term in brackets in the exponent of Eqs. 5-7 is the Damkohler number. The length term in the Damkohler number is scaled to the length of the core, and the simulated injection rate was the same as that in the experiment. Actual values of the diffusion rate, viscosity, acid concentration, and stoichiometric coefficient were used. The three expressions essentially differ only in the functionality of the bond diameter. Model results using the two expressions for the transport-limited case differ very little. The effects of kinetic as opposed to transport limitations are discussed below with the simulation results.

The growth rate of each bond is assumed to be uniform along the length of the bond, to be proportional to the total rate of acid consumption per unit length of bond, and to be quasisteady with respect to the acid consumption. These assumptions, combined with Eq. 6, for example, give the overall growth in diameter in 
an individual bond at any time $t$ :

$$
\begin{array}{r}
\Delta D_{i} / D_{i}=\left(C o_{i} / \gamma\right)\left(Q_{i} \Delta t / D_{i}^{2} L_{i}\right) \\
\cdot\left\{1-\exp \left[-a\left(D_{e} L_{i} / Q_{i}\right)^{b}\right]\right\}
\end{array}
$$

The growth rate thus depends on a stoichiometric term describing the amount of solid consumed per unit volume of reactant, a dimensionless time or space time, and the Damkohler number. Changes in the bond diameters cause a redistribution of the flow throughout the network which is monitored as a function of time.

\section{Channeling in the network}

Owing to the distribution of resistances, a distribution of fiow paths will exist, and flow along certain pathways through the network will be higher than in neighboring paths. Paths with greater flow grow preferentially over their neighbors because they receive more acid, which is carried further into the lattice along these paths before being consumed. This in turn further reduces the resistance to flow in these paths and eventually one or more channels completely dominates and carries virtually all the flow.

Information predicted by the simulation includes the structure of channels and the rate of channel propagation as a function of the fluid flow rate, the reaction rate, the acid concentration, and the original permeability and heterogeneity of the media. The pressure drop (or flow rate in the case of a constantpressure boundary condition) is determined as a function of dimensionless time. Perhaps most important, information about the structure of the channels that form is obtained by drawing the dissolved network such that the width of each bond is a function of the amount of material dissolved in that bond. The channel structure predicted by the simulation is shown in Figures 12 and 13. The size of the triangular network used was $30 \times 50$

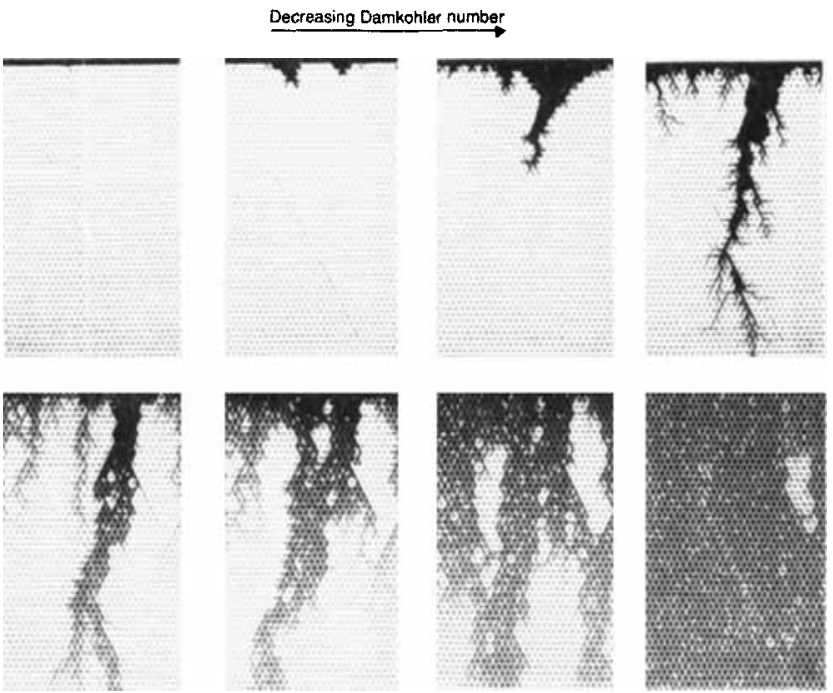

Figure 12. Simulation results showing channeling in network for transport-limited case, $\mathrm{Da} \alpha \mathrm{cL} / \mathrm{O}$, $c=$ constant.

The Damkohler numbers for the run and the final permeability ratios are from top left down, respectively, 12.68/1.06, 5.72/ $1.308,1.23 / 243,0.265 / 952,0.0572 / 3,650$, and $0.0123 / 7,433$

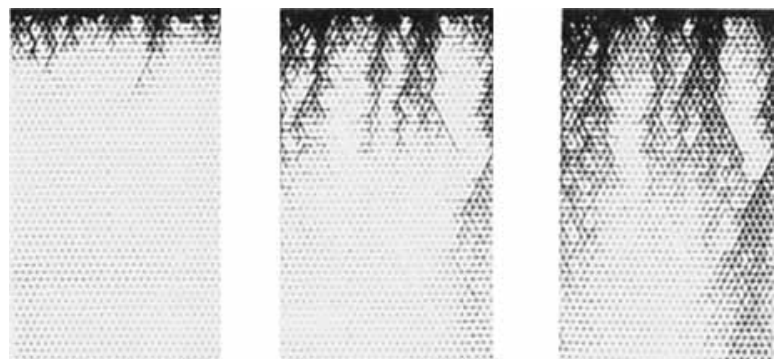

Figure 13. Simulation results showing channeling in the network for the kinetic rate-limited case, Da $\alpha$ $c D L / Q$.

The Damkohler numbers for the run and the final permeability ratio are from top, respectively, $0.0572 / 2.601,0273 / 13.58$, and $0.0123 / 99.77$

nodes, and the results were not sensitive to the network size when perturbed around this case. Several realizations carried out for certain cases confirmed that there were no significant effects on the channel structure over the entire range of the Damkohler number. For different realizations the simulated channel structures, as in Figures 12 and 13, are of course slightly different but have the same characteristics; examples of multiple realizations and other details are presented by Hoefner (1987). Information regarding the channel structure is very useful in understanding the effects of channel structure on the increase in permeability.

\section{Mass-transfer limited regime}

A number of realizations were carried out to study the relative effects of mass transfer and flow (i.e., the Damkohler number.) In order to compare the experimental observations with the channel structure predicted by the simulation, the amount of material dissolved from each bond in the network was determined. To quantify the channel structure the width of each bond was drawn, Figure 12, such that it was proportional to the square root of the amount of material dissolved from that bond. A series of networks which show the variation of channel structure with Damkohler number are presented in Figure 12. Here the Damkohler number is varied over a range of two-and-onehalf orders of magnitude, where the Damkohler number is defined as;

$$
D a=a D_{e}^{2 / 3} L_{i} / Q_{i}
$$

At high values of $D a$, corresponding to large dissolution rates or low injection rates, we see that acid penetrates only a short distance into the network. Virtually all of the reactant is consumed in the first few bonds encountered, analogous to the case of complete acid spending on the inlet face of a core, as in Figure 6. At lower values of the Damkohler number, which can result either from increasing the injection rate or decreasing the reaction rate, channeling starts to occur. Over the middle range of the Damkohler numbers, the channeling behavior is obvious. Furthermore, as the Damkohler number is decreased the channel becomes more branched, which is exactly as observed in the experiments, Figure 5. At extremely low Damkohler numbers, 
corresponding to very low dissolution rates relative to acid flow rates, very little acid spending occurs and an extremely uniform dissolution takes place throughout the majority of pore space. This prediction is analogous to the very fine network of channels observed in the Wood's metal castings at low Damkohler numbers.

Depending on the particular solution or correlation for the mass transfer coefficient, the diameter (and flow rate) dependence of the Damkohler number may differ slightly from that in Eq. 9. The Damkohler number can range from being proportional to bond diameter to the zero power to about the minus one-half power in the transport-limited regime. The effect of increasing the diameter dependence in the denominator of the Damkohler number is to reduce the rate of reactant consumption in the bonds as they grow. When this happens, channeling is favored and the range of the Damkohler number over which channeling occurs increases. The network simulations shown in Figure 16 correspond to approximately the same range of $D a$ as the castings shown in Figure 5, and so the zero power of the diameter, Eq. 9, is appropriate to simulate channeling in this system.

\section{Reaction-rate limited regime}

The variation of the channel structure in the reaction-rate limited regime is shown by a series of networks in Figure 13. The reaction in this case is slow, and the result is a more uniform acid attack. Dominant channels are less likely to form, as a result of the diameter dependence of the expression for acid consumption.

The range of the Damkohler number over which channeling can occur in the reaction-rate limited case is very small. The Damkohler number for the reaction-rate limited regime is defined as $D a=a k L_{i} D_{i} / Q_{i}$. Since the Damkohler number in this case is proportional to the bond diameter to the first power, the rate of reaction in each bond increases dramatically as the bond grows. Thus the rate of reactant consumption increases in any dominant channels that start to form, and this slows the rate of propagation of the reaction front. In the experiments, frontal propagation is much slower in the dolomite (reaction-rate limited dissolution) than in the limestone (transport-limited dissolution).

Depending on the rate-limiting regime considered, the diameter dependence of the Damkohler number can range from +1 to $-1 / 2$. Whenever the rate of reactant consumption increases with increasing bond diameter, channeling is inhibited and the frontal propagation will be slow. When the diameter dependence is negative, the rate of reactant consumption decreases as the channels grow, and the formation of channels is very favorable. Decreasing the diameter dependence from positive to negative increases the range of $D a$ over which channeling can occur, and for the transport-limited case, $D a$ proportional to $D^{O}$ simulates the experimental data for Indiana limestone very well.

The permeability response of the network as a function of Damkohler number is shown in Figure 14. The curves show the same characteristic shape as those observed in the experiments, where the increase in the permeability is very little until acid breakthrough, at which point there is a sudden and dramatic permeability increase. The volume of fluid required to achieve a specific increase in the permeability ratio decreases with decreasing $D a$ for the transport-limited case, and increases with

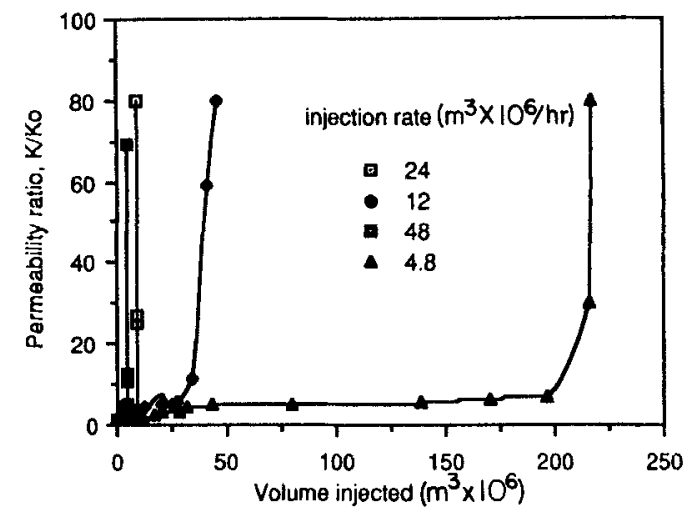

Figure 14. Typical network permeability response as a function of simulated volume of fluid injected.

decreasing $D a$ for the reaction-limited case. These general trends agree with the experimental observations.

\section{Channel branching and effect on growth rate}

A porous medium is made up of many interconnected pores, and branching on the pore level is due to the effects of dissolution on the distribution of flow through pore connections. To elucidate this effect, a simple $\mathrm{Y}$-branched tube, or equivalently, parallel flow through two cylindrical tubes is analyzed. This is shown schematically in Figure 15. For a fixed pressure drop, the relative flow rates through the parallel cylindrical tubes are a function of the diameters of the tubes. As dissolution proceeds, the branches grow in diameter and the flow redistributes itself in a manner that depends on the relative growth rates of the two branches. More rapid growth of the smaller branch will result in an increase in its fraction of the total flow.

The ratio of the diameters of two cylindrical channels are shown as a function of dimensionless time in Figure 16. The effect of the rate of growth on this diameter ratio is shown for a kinetically rate-limited reaction and for a diffusion-limited reaction. For the diffusion-limited case, the rate of transport of reactant is described by the Graetz expression discussed earlier. When the reaction -is fast, such that virtually no unconsumed acid exits the channels, the amount of material removed from each channel is given by $Q_{i} \mathrm{Co}_{i} \Delta t / \gamma$. Since the flow distribution is proportional to the diameters to the fourth power, the diameter of the larger pore will grow very fast relative to the diameter

Flow and Dissolution:

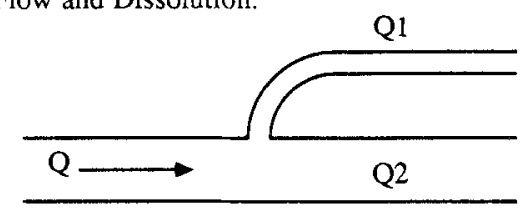

$$
\begin{aligned}
& \text { High reaction rate: } Q 1 \text { increases relative } \\
& \text { to } Q 2 \text { (inhibits branching). }
\end{aligned}
$$

Figure 15. Cylindrical tube concepts for branching on pore level.

Fluid diverges at the fork, and the two branches of the system then grow at rates that are a function of the Damkohler number 


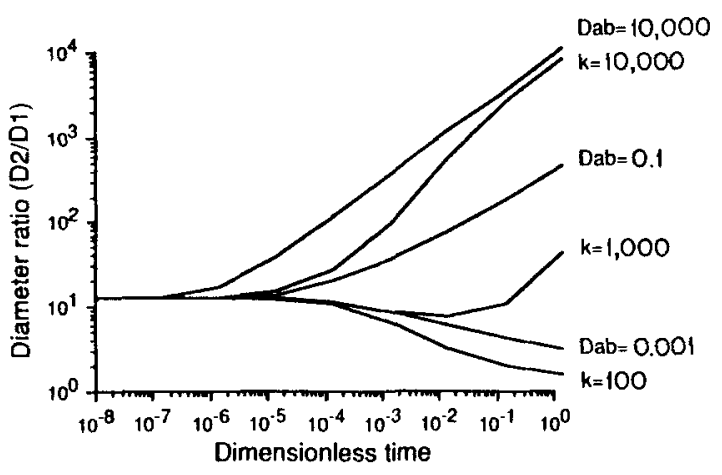

Figure 16. Simulation results for a simple tube model of pore-level channel branching.

At high Damkohler number, (i.e., high values of diffusion or reaction rate constant) the larger branch dominates, inhibiting branching; at low Damkohler number the size of the branches tend to become equal, and this favors branching

of the smaller pore. The larger pore will eventually carry virtually all of the flow, and this is what is observed in Figure 16. Such a condition inhibits branching as smaller pathways tend to die out in favor of the more dominant ones.

If, on the other hand, the net reaction rate is relatively slow, such that the exiting acid concentration is virtually equal to that entering, then the ratio of the diameters tends toward unity, also shown in Figure 16, and the flow rates tend to equalize in the two tubes. This condition favors branching because flow paths, which initially varied widely in size, tend to converge toward a common size. The various pathways then grow at approximately equal rates.

Branching on the pore level is important in understanding microscopic channel structure. The behavior of longer channels, however, is of greater interest because these ultimately control the process of interest. Furthermore, different factors affect branching on longer length scales than branching on the pore scale. For the case of longer branches, the flow in one path will be affected by the presence of neighboring paths because of pore connectedness. Also, the propagation of an individual pathway is not determined by the resistance in that pathway, as on the pore scale. Rather, the relative flow rates in the longer branches are a function of the pressures at all points surrounding the channel. The relative propagation rates are thus determined by the number of paths, the length of a path in relation to those nearby, and the proximity of the channels to one another. This is true of two branches diverging from a point, and also of two channels forming on the inlet flow face some distance apart.

The network simulation was used to study the effects of competition between two channels. The network was first decorated with a monodispersed distribution of bond resistances, and then two dominant pathways were added; this is shown schematically in Figure 17. The flow through these channels is calculated as a function of the distance between the channels and of the difference in the channel lengths. The networks used were very long relative to the lengths of the channels, thus simulating a semiinfinite medium.

Competing branch results are shown in Figure 18, where the Damkohler numbers (essentially the flow rates) in the channels are compared as a function of the length difference and the distance between the channels. One observes from this figure that as the difference in the length of the two channels increases or as

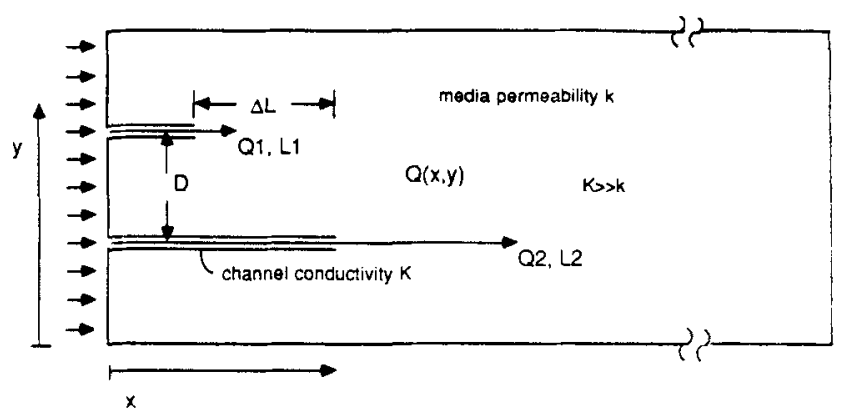

Figure 17. Cylindrical tube concepts for branching on length scales longer than pores.

Fluid flows through these channels in parallel; the relative lengths of the two channels and the distance between them affects the relative flow rates through the two pathways

the distance between the channels decreases, flow becomes dominant in the longer branch. The flow rate in the shorter branch becomes so small that this branch will cease to propagate.

These results show that the difference in the lengths of two channels is very unlikely to exceed the distance between the channels. This concept is consistent with experimental observation that a branch from a dominant channel never grows much longer than the distance between the branch and the main channel. This result is important when trying to predict the expected density of wormholes on the inlet flow face of a core, for example. In a linear coreflood, the number of wormholes that are expected to propagate to a significant fraction of the total length of the core should be roughly equal to the ratio of the inlet area to the core length. In a radial coreflood, this means that no more than four or five channels will form in a plane, and this is what is observed in all radial DLA-type patterns.

\section{Summary}

The network model presented here accounts for every characteristic of the experimental coreflood data, both in permeability response and channel structure. The unique feature of the network modeling approach is the ability to account for the effects

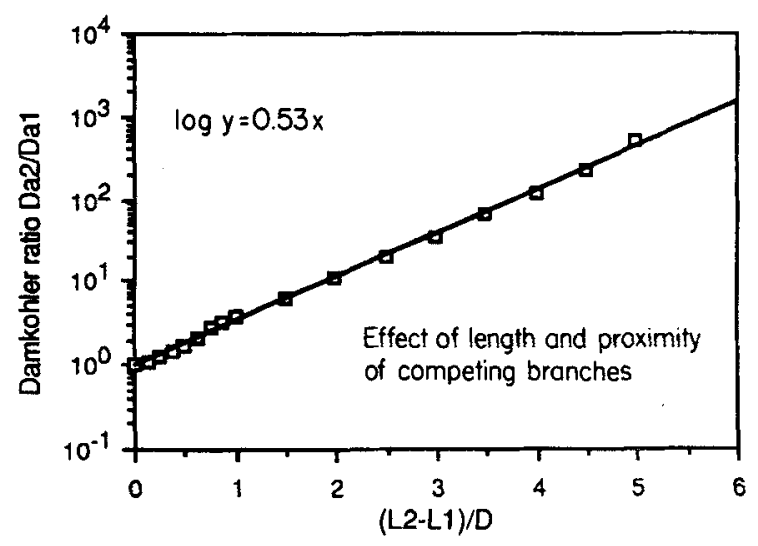

Figure 18. Simulation results for a simple tube model of channel branching on longer length scales. When the difference in the length of two pathways exceeds the distance between the pathways, the flow in the shorter channel is reduced substantially, and that pathway will die out 
of channel branching on the rate of propagation of the reaction front.

Analysis of the photos of channel castings shows that the pore evolution process is controlled by the Damkohler number for flow and reaction. When the Damkohler number is large, the effect of increasing the injection rate or decreasing the reaction rate (decreasing $D a$ ) is to increase stimulation efficiency. At smaller values of the Damkohler number, flow channels become more highly branched, tend to be finer, are smaller in diameter, and permeate the medium more uniformly. In the limit of very small values of $D a$, a very highly branched network of channels forms, and the result is dissolution over a high mineral surface area. Extreme channel branching causes stimulation efficiency to decrease with decreasing values of the Damkohler number. On the pore level, small values of the Damkohler number favor a more uniform growth of many small pathways, and a more highly branched channel structure results.

\section{Notation}

$a, b=$ constants

$C o_{i}=$ inlet reactant concentration in bond $i$, mole reactant/length cubed reactant

$D a b, D_{e}=$ effective diffusion coefficient, mass/length squared time

$D_{i}=$ diameter of bond $i$, length

$k=$ surface reaction rate constant

$L_{i}=$ length of bond $i$, length

$Q_{i}=$ volumetric flow rate in bond $i$, length cubed $/$ time

$t=$ time

$v=$ kinematic viscosity

$\gamma=$ stoichiometric coefficient, mole reactant/length cubed rock

\section{Literature Cited}

Chen, J. D., and D. Wilkinson, "Pore-Scale Viscous Fingering in Porous Media," Phys. Rev. Lett., 47(18), 1892 (1985).

Daccord, G., "Chemical Dissolution of a Porous Medium by a Reactive Fluid," Phys. Rev. Lett., 58, 479 (1987).

Daccord, G., and R. Lenormand, "Fractal Patterns from Chemical Dissolution," Nature, (1986).

Daccord, G., J. Nittmann, and M. E. Stanley, "Radial Viscous Fingering and Diffusion-Limited Aggregation: Fractal Dimension and Growth Sites," Phys. Rev. Lett., 56(4), 336 (1985).

Fatt, 1., "The Network Model of Porous Media. I, II, III.," Pet. Trans. AIME, 207, 144 (1956).

Guin, J. A., R. S. Schechter, and 1. H. Silberberg, "Chemically Induced Changes in Porous Media," Ind. Eng. Chem. Fundam., 10(1), 50 (1971).

Hoefner, M. L., "Matrix Acidizing in Carbonates Using Microemulsions: The Study of Flow, Dissolution and Channeling in Porous Media," Ph.D. Thesis, Univ. Michigan, Ann Arbor (1987).

Hoefner, M. L., and H. S. Fogler, "Effective Matrix Acidizing in Carbonates Using Microemulsions," Chem. Eng. Prog., 40 (Apr., 1985).
- "Effect of Acid Diffusion in Matrix Acidizing in Carbonates," J. Pet. Tech. (1987).

Jakob, M., Heat Transfer, I, Wiley, New York 451 (1949).

Jerauld, G. R., J. C. Hatfield, L. E. Scriven, and H. T. Davis, "Percolation and Conduction on Voronoi and Triangular Networks: A Case Study in Topological Disorder," J. Phys. Chem. 17, 3429 (1984).

Lapidus, G. R., A. M. Lane, K. M. Ng, and W. C. Connor, "Interpretation of Mercury Porosimetry Data Using a Pore-Throat Network Model," Chem. Eng. Commun., 38, 33 (1985).

Lund, K., H. S. Fogler, and C. C. McCune, "Acidization. I: The Dissolution of Dolomite in Hydrochloric Acid," Chem. Eng. Sci., 28, 691 (1973).

Lund, K., H. S. Fogler, C. C. McCune, and J. W. Ault, "Acidization. II: The Dissolution of Calcite in Hydrochloric Acid," Chem. Eng. Sci., 30, 825 (1975).

McCune, C. C., H. S. Fogler, and W. E. Kline, "An Experimental Technique for Obtaining Permeability-Porosity Relationships in Acidized Porous Media," Ind. Eng. Chem. Fundam., 18(2), 188 (1979).

Meakin, P., "Diffusion-Controlled Cluster Formation in 2, 3, and 4 Dimensions," Phys. Rev. A., 27, 604 (1983).

Nierode, D. E., and B. B. Williams, "Characteristics of Acid Reaction in Limestone Formations," Soc. Pet. Eng. J., 407 (Dec., 1971).

Nougaro, J., and C. Labbe, "Etude des Lois de L'Acidification dans le Cas d'un Calcaire Vacuolaire," Rev. Fran. Pet., 10(5), 354 (1955).

Paterson, L." "Diffusion-Limited Aggregation and Two-Fluid Displacements in Porous Media," Phys. Rev. Lett., 52(18), 1621 (1984).

Rose, W., "Studies of Waterflood Performance. III: Use of Network Models, Ill.," St. Geolog. Surv. Circ., 237, 1 (1957).

Rowan, G., "Theory of Acid Treatment of Limestone Formations," $J$. Inst. Pet., 45(431), 321 (Nov., 1959).

Sahimi, M., H. T. Davis, and L. E. Scriven, "Dispersion in Disordered Porous Media," Chem. Eng. Commun., 23, 329 (1985).

Sahimi, M., B. D. Hughes, L. E. Scriven, and H. T. Davis, "Dispersion in Flow Through Porous Media. I: One-Phase Flow," Chem. Eng. Sci., 41, 2103 (1986a).

Sahimi, M., A. A. Heida, H. T. Davis, and L. E. Scriven, "Dispersion in Flow Through Porous Media. II: Two-Phase Flow," Chem. Eng. Sci., 41, 2123 (1986b).

Simon, R., and F. J. Kelsey, "The Use of Capillary Tube Networks in Reservoir Performance Studies. I, II," Soc. Pet. Eng. J., 99, 345 (Aug., 1972).

Wardlaw, N. C., and R. P. Taylor, "Mercury Capillary Pressure Curves and the Interpretation of Pore Structure and Capillary Behaviour in Reservoir Rocks," Bull. Can. Pet. Geo., 24(2), 225 (June, 1976).

Witten, T. A., and L. M. Sander, "Diffusion-Limited Aggregation, A Kinetic Critical Phenomenon," Phys. Rev. Lett., 47, 1400 (1981). (1983).

\section{Acknowledgment}

We would like to acknowledge NSF Grant No. CBT-8516458 and CPE-8108197 for the support of this work.

Manuscript received Feb. 12, 1987 and revision received Aug. 25, 1987. 